\title{
Commentary: A Neural Basis for the Acquired Capacity for Suicide
}

\begin{abstract}
Xenia Gonda ${ }^{1,2,3,4 *}$
${ }^{1}$ Department of Psychiatry and Psychotherapy, Semmelweis University, Budapest, Hungary, ${ }^{2}$ MTA-SE

Neuropsychopharmacology and Neurochemistry Research Group of the Hungarian Academy of Science, Semmelweis University, Budapest, Hungary, ${ }^{3}$ NAP-A-SE New Antidepressant Target Research Group, Semmelweis University,

Budapest, Hungary, ${ }^{4}$ Laboratory for Suicide Prevention and Research, National Institute for Psychiatry and Addictions, Budapest, Hungary
\end{abstract}

Keywords: suicide, gender, neurobiology, imaging, completed suicide

\section{A commentary on}

A Neural Basis for the Acquired Capability for Suicide

by Deshpande G, Baxi M, Witte T, Robinson JL. Front Psychiatry (2016) 7:125. doi:10.3389/ fpsyt.2016.00125

Every year suicide accounts for nearly $2 \%$ or altogether one million deaths, one happening approximately every $40 \mathrm{~s}$ (1). Completed suicide happens significantly more frequently in males in all countries except for rural China (2), and in several countries, it is the leading cause of death in males younger than 40 years of age and is also among the leading causes in other age groups (3). In spite of this significant burden, our understanding of suicide is far from complete. Suicide is a complex phenomenon, arising in almost $90 \%$ in the framework of psychiatric disorders, mostly associated with affective illness. However, the lack of sufficient understanding of both psychosocial and neuroanatomical contributors and comprehensive biopsychosocial models leaves a huge gap in identifying targets for the early prediction, screening, detection, and intervention in case of suicidal behavior.

As mentioned earlier, completed suicide rates are much higher in men compared to women especially in the young age groups. Therefore, understanding the neurobiological background of the gender difference would be a promising approach for better understanding of the occurrence of this phenomenon and can also give biological support to psychosocial theories which in turn would also utilize biological findings. Although the most frequent precursor of suicide is major depression, which is three times more common in reproductive age women compared to men, males are paradoxically markedly overrepresented among suicide victims. Several clinical correlates of more common suicidal mortality can be identified in men including employment of more highly lethal methods, comorbid alcohol and substance use, less common help-seeking behavior, and increased impulsive aggressive traits (4). These are coupled with poorer treatment compliance and worse response to certain antidepressants in men further hindering recognition and prevention of processes leading to suicide in men (2). Therefore, identification of gender-specific factors in the emergence of suicide needs to be identified to design-specific interventions. Furthermore, understanding the role of gender in differences in the manifestation of behaviors along the suicide spectrum would also yield important new insight concerning the nature of suicide in general.

There are important gender differences in brain processes involved in learning, memory, language, fear, and anxiety as well as significant gender differences in the prevalence rates of several neuropsychiatric disorders, which may result from sex chromosome effects, developmental hormonal effects, or hormonal modulations during adulthood (5) and may also be consequences of gender-related psychosocial effects. So far, gender differences in dopamine and serotonin functions and genetics (6) and associations between cortisol and male suicide have been reported $(7,8)$. Testosterone also plays an important role in the gender effect observable in suicide, and this effect may also interact 
with age (9). Urocortin and BDNF also appear to play a genderspecific role in male suicidal behavior (10), and interestingly there appears to be a gender difference in the putative antisuicidal effect of lithium as well (11). This already complex picture suggests that the investigation of genetic and biological factors in the background of suicide should be conducted from a gender perspective.

In line with this previous knowledge, the present paper (12) focuses on understanding the neural substrates in the background of gender differences in completed suicide by investigating a potential neural network for acquired capability for suicide (ACS), which includes emotional stoicism, sensation-seeking, pain tolerance, fearlessness of death, thus involving neurobiological structures and processes associated with various endophenotypes of suicidal behavior outlined using activation likelihood estimation meta-analysis. The authors report that male-specific neural ACS networks are both more widespread and more diverse compared to female-specific networks and are dominated by motor regions, while female networks show a dominance of limbic regions, explaining why suicidal behavior is manifested more on the action and motor level in males and on the emotional and ideation level in females.

One important aspect of this paper is that while most imaging studies report on suicide attempts, this is one of the rare studies focusing on imaging methodology in completed suicide. As gender shows an inverse association with attempted vs. completed

\section{REFERENCES}

1. World Health Organisation. Preventing Suicide, a Global Imperative (2014). Available from: http://www.who.int/mental_health/suicide-prevention/ world_report_2014/en/

2. Hawton K. Sex and suicide - gender differences in suicidal behaviour. $\mathrm{Br}$ J Psychiatry (2000) 177:484-5. doi:10.1192/bjp.177.6.484

3. Van Heeringen K, Bijttebier S, Desmyter S, Vervaet M, Baeken C. Is there a neuroanatomical basis of the vulnerability to suicidal behavior? A coordinate-based meta-analysis of structural and functional MRI studies. Front Hum Neurosci (2014) 22(8):824. doi:10.3389/ fnhum.2014.00824

4. Rutz W, Vonknorring L, Pihlgren H, Rihmer Z, Walinder J. Prevention of male suicides - lessons from Gotland Study. Lancet (1995) 345(8948):524-524. doi:10.1016/S0140-6736(95)90622-3

5. McCarthy MM, Arnold AP, Ball GF, Blaustein JD, De Vries GJ. Sex differences in the brain: the not so inconvenient truth. J Neurosci (2012) 32(7):2241-7. doi:10.1523/Jneurosci.5372-11.2012

6. Huang YY, Cate SP, Battistuzzi C, Oquendo MA, Brent D, Mann JJ. An association between a functional polymorphism in the monoamine oxidase A gene promoter, impulsive traits and early abuse experiences. Neuropsychopharmacology (2004) 29(8):1498-505. doi:10.1038/ sj.npp. 1300455

7. Jokinen J, Nordstrom AL, Nordstrom P. CSF 5-HIAA and DST non-suppression - orthogonal biologic risk factors for suicide in male mood disorder inpatients. Psychiatry Res (2009) 165(1-2):96-102. doi:10.1016/j. psychres.2007.10.007 suicide, studies on suicide attempters are unable to address the question of gender sufficiently. Furthermore, the current study uses a psychosocial theory and psychological constructs as a basis for neurobiological research for a comprehensive approach. The results also validate the construct of ACS which may be a key and potentially operationalizable construct in suicidal behavior, by identifying its underlying neural substrates.

This research indeed provides us with important new data on the gender differences in completed suicide; however, the applicability of its method and approach goes way beyond that. Rather than focusing on suicidal behavior as a single entity, or on suicide-related single individual endophenotypes such as impulsiveness or hopelessness, this paper with a unique novel approach targets a complex pathway of behaviors leading to the emerging risk for suicide. Thus, these novel results not only provide a new insight into the gender differences in fatal suicides but also set an example to the complex approach of this complex phenomenon, which may pave the way for the development of more detailed and accurate dynamic models in the understanding of suicidal behavior helping to identify targets for prediction and intervention in both genders.

\section{AUTHOR CONTRIBUTIONS}

XG undertook a review of the literature, conceived this general commentary, and wrote and reviewed all drafts.

8. Ghaziuddin N, King CA, Welch K, Ghaziuddin M. Depressed suicidal adolescent males have an altered cortisol response to a pharmacological challenge. Asian J Psychiatr (2014) 11:13-9. doi:10.1016/j.ajp.2013.10.005

9. Sher L. High and low testosterone levels may be associated with suicidal behavior in young and older men, respectively. Aust N Z J Psychiatry (2013) 47(5):492-3. doi:10.1177/0004867412463976

10. Kozicz T, Tilburg-Ouwens D, Faludi G, Palkovits M, Roubos E. Genderrelated urocortin 1 and brain-derived neurotrophic factor expression in the adult human midbrain of suicide victims with major depression. Neuroscience (2008) 152(4):1015-23. doi:10.1016/j.neuroscience.2007.12.050

11. Ishii N, Terao T, Araki Y, Kohno K, Mizokami Y, Shiotsuki I, et al. Low risk of male suicide and lithium in drinking water. J Clin Psychiatry (2015) 76(3):319-26. doi:10.4088/JCP.14m09218

12. Deshpande G, Baxi M, Witte T, Robinson JL. A neural basis for the acquired capacity for suicide. Front Psychiatry (2016) 7:125. doi:10.3389/ fpsyt.2016.00125

Conflict of Interest Statement: The author declares that the research was conducted in the absence of any commercial or financial relationships that could be construed as a potential conflict of interest.

Copyright (C) 2017 Gonda. This is an open-access article distributed under the terms of the Creative Commons Attribution License (CC BY). The use, distribution or reproduction in other forums is permitted, provided the original author(s) or licensor are credited and that the original publication in this journal is cited, in accordance with accepted academic practice. No use, distribution or reproduction is permitted which does not comply with these terms. 